\title{
The Chandrasekhar limit for quark stars
}

\author{
Shibaji Banerjee円, Sanjay K. Ghosh[ and Sibaji Raha \\ Department of Physics, Bose Institute, \\ 93/1, A. P. C. Road, Calcutta 700 009, INDIA
}

\begin{abstract}
The Chandrasekhar limit for quark stars is evaluated from simple energy balance relations, as proposed by Landau for white dwarfs or neutron stars. It has been found that the limit for quark stars depends on, in addition to the fundamental constants, the Bag constant.
\end{abstract}

Compact objects like the white dwarfs and neutron stars have been a topic of interest for several decades. These objects are produced as the end product of the stellar evolution, i.e., when the nuclear fuel of the normal stars have been consumed. White dwarfs are supported against gravitational collapse by the degeneracy pressure of electrons whereas for neutron stars, this pressure comes mainly from the degenerate neutrons. It is well known that both these classes of collapsed stars have a maximum mass $\left(\sim 1.5 M_{\odot}\right)$, beyond which

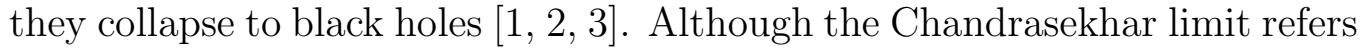
strictly to white dwarfs, the limiting mass for neutron stars is also loosely called the Chandrasekhar limit, primarily because the limits in the two cases turn out to be the same [2].

The underlying quark structure of the nucleons suggests a possible hadron - quark phase transition at high density and/or temperature. This, in turn, implies the possible existence of another kind of compact objects, namely

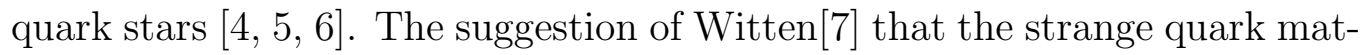
ter may be the true ground state of the strongly interacting matter started a new era in this subject. In this circumstance, quark stars, if they are formed, would preferably convert to strange stars, comprising $u, d$ and $s$ quarks, under weak interaction. Several authors ( for example, [8, 9] ) have used different models to understand the properties of strange stars. For a review, see [6].

The unique feature of strange stars, which separates them from other compact objects, is that these need not be the direct product of stellar evolution. These are most likely to be produced due to the hadron quark phase

\footnotetext{
${ }^{1}$ email: phys@bosemain.boseinst.ernet.in

${ }^{2}$ email: phys@bosemain.boseinst.ernet.in

${ }^{3}$ email: sibaji@bosemain.boseinst.ernet.in
} 
transition inside the neutron stars as the density is high enough to favor such a transition. For such quark stars, the maximum mass would indeed be almost the same as that for neutron stars. On the other hand, it is also conceivable that if a large amount of quark matter exists in the universe as a relic of the cosmological quark-hadron phase transition [10], it could clump under gravitational interaction and even form invisible quark galaxies [11]. The 'stars' of such a galaxy would be strange stars which do not evolve from neutron stars and thus are not governed by the Chandrasekhar limit for neutron stars. It is therefore most pertient to ask if there exists, just like the case of ordinary compact stars, an upper limit on the strange stars beyond which they would be gravitationally unstable. Starting with the seminal work of Witten [7], most authors have concentrated on solving the TolmanOppenheimer-Volkov (TOV) equation (see, for example, [3]) numerically for the quark matter equation of state. While the results show that indeed there exists a limiting mass for quark stars (which is very close to that for neutron stars), there is no a priori argument to prove that such a limit should exist or that it should depend mostly on fundamental constants, as is the case for the ordinary compact stars [3]. Our aim in this letter is to show analytically, from first principles, that such a limit exists for compact quark stars and that it is indeed determined mostly by universal constants.

We start with the most general and simple picture of energy balance, proposed by Landau [2]. The strange star is composed of a 3 flavour system of massless quarks, confined in a large bag [⿴囗十 characterized by a constant energy density $B$. As in the case of white dwarfs and neutron stars, the equilibrium should occur at a minimum of the total energy per fermion $e$, where $e \equiv$ $e_{F}+e_{G}$, in which $e_{F}$ is the fermi energy and $e_{G}$ is the gravitational energy per fermion. The crucial difference between the cases of quark stars and the ordinary compact stars lies in the estimation of the (Newtonian) gravitational energy, considered to be a macroscopic quantity. For ordinary compact stars, this mass is due almost entirely to the baryons. For quark stars, however, the total mass is the total ( thermodynamic as well as the confining ) energy in the star. For the purpose of estimating the gravitational energy per fermion, one can then define an effective quark mass which incorporates both these contributions. A suitable prescription for such a definition was formulated quite some time ago [12.

The number density of fermions is related to the chemical potential as

$$
n=\frac{g}{6 \pi^{2}} \mu^{3}
$$

which dictates that 


$$
\mu=\left(\frac{9 \pi}{2 g}\right)^{\frac{1}{3}} \frac{N^{\frac{1}{3}}}{R}
$$

In the above relations, $n$ is the number density, $N$ the total number of fermions in a star of radius $R, g$ the statistical degeneracy factor and $\mu$ is the chemical potential.

The fermion energy density is given by

$$
\varepsilon_{F}=\frac{g}{8 \pi^{2}} \mu^{4}
$$

and hence the fermi energy per particle of the quarks becomes

$$
e_{F}=\frac{\varepsilon_{F}}{n}=\frac{3}{4}\left(\frac{9 \pi}{2 g}\right)^{\frac{1}{3}} \frac{N^{\frac{1}{3}}}{R}
$$

The mass $M$ of the star can be written in terms of $N$ and $B$ (the bag constant), if the density $\rho(r)$ in the star is assumed to be roughly constant throughout the volume of the star. Hence using eq.(3),

$$
M=\int_{0}^{R} 4 \pi r^{2} \rho(r) d r=\frac{4}{3} \pi R^{3} B+e_{F} N=\frac{3}{4}\left(\frac{9 \pi}{2 g}\right)^{\frac{1}{3}} \frac{N^{\frac{4}{3}}}{R}+\frac{4}{3} \pi B R^{3}
$$

Extremising the mass $M$ (eq. (4) with respect to $R$ gives,

$$
\left(\frac{9 \pi}{2 g}\right)^{\frac{1}{3}} \frac{N^{\frac{4}{3}}}{R^{4}}=\frac{16}{3} \pi B \Rightarrow \varepsilon_{F}=3 B
$$

Substituting eq.(5) in the expression for $M$ (eq. 4),

$$
M=4 B V=\frac{16}{3} \pi B R^{3}
$$

Note that this is very similar to the condition obtained for hadronic bags [13. The task at hand then is to find the $R$ for which the total energy per fermion would be maximum.

The gravitational energy per fermion $e_{G}$ is

$$
e_{G}=-\frac{G M m_{e f f}}{R}
$$

where $m_{\text {eff }}$ is the effective quark mass inside the star. Assuming that the effective quark mass contributes to the total star mass $M$, one can write for a strange star with $N$ quarks,

$$
M=N m_{e f f} \Rightarrow m_{e f f}=\frac{4 B}{n}
$$


As mentioned above, the effect of confinement in a quark matter system was shown [12 to be incorporable in the effective quark mass, which, the quarks being fermions, coincides with the quark chemical potential. As a result, one gets, in the limit of vanishing quark density [12],

$$
\mu=\frac{B}{n}
$$

- This, together with the eq.(8), gives

$$
m_{e f f}=4 \mu
$$

where all the energy ( thermodynamic and confining ) is included in the effective gravitational mass of the quarks inside the strange star.

Using equations (7), (8) and (9) we get

$$
e_{G}=-\frac{64}{3}\left(\frac{9 \pi}{2 g}\right)^{\frac{1}{3}} G \pi B R N^{\frac{1}{3}}
$$

Minimising the total energy $e=e_{F}+e_{G}$ with respect to $N$, we get the expression for maximum value of $R$ as

$$
R_{\max }=\frac{3}{16} \frac{1}{\sqrt{\pi G B}}
$$

Finally, the maximum mass of the strange star is computed by substituting the value of $R_{\max }$ (from equation 11) in equation ( 6).

$$
M_{\max }=\frac{16}{3} \pi B R_{\max }^{3}
$$

The chemical potential $\mu$ can be evaluated in terms of $B$ using equations (2) and (8). Substituting this in eq.(8) gives the value of $N_{\max }$. The values of $R_{\max }, M_{\max }$ and $N_{\max }$ are tabulated below for various values of the Bag constant $B$.

\begin{tabular}{|c|c|c|c|}
\hline$B^{1 / 4}(\mathrm{MeV})$ & $R_{\max }(\mathrm{Km})$ & $\frac{M_{\max }}{M_{\odot}}$ & $N_{\max }$ \\
\hline \hline 145 & 12.11 & 1.54 & $1.55 \times 10^{57}$ \\
\hline 200 & 6.36 & 0.81 & $5.90 \times 10^{56}$ \\
\hline 245 & 4.24 & 0.54 & $3.21 \times 10^{56}$ \\
\hline
\end{tabular}

We thus find that even for quark stars, there does exist a limiting mass, the so-called Chandrasekhar limit, which is mostly determined by the universal constants ( $G$ as well as $\hbar$ and $c$, which do not occur explicitly due to our use of the naturalised units ) and the Bag energy B. Although treated as a parameter here, the Bag energy too has, roughly, the status of another 
universal constant, being the difference between the non-perturbative and the perturbative vacua of Quantum Chromodynamics. The physical radius $R_{\max }$, corresponding to the maximum mass as well as the maximum mass itself, are independent of the number of flavours, as seen from equations (11, 12). Although $N_{\max }$ depends on the statistical degeneracy factor $g$ ( or equivalently, the number of flavours), the dependence is extremely weak, as can be readily checked from equation (11). In fact, we have verified that there is almost no difference in $N_{\max }$ between the cases with $g=2$ and 3 . This, in turn, implies that the assumption of massless quarks (even for $s$ quarks ) does not affect these results.

Admittedly, our purpose here has been mostly illustrative. Nevertheless, these limits agree very well with those found in the numerical solutions of the TOV equation (see, for example, [0] ). Even under the simplifying assumption of a constant density profile, necessary for an analytical solution, the scaling

behavior $\left(R_{\max } \propto B^{-1 / 2}, M_{\max } \propto B^{-1 / 2}\right.$, obtained from the numerical solutions ) hold true. This proves that the simple picture presented here adequately incorporates the essential physics.

To conclude, we have shown that there exists a limiting mass (the Chandrasekhar limit) for compact quark stars, beyond which they would be gravitationally unstable. As with white dwarfs and neutron stars, this mass depends mostly on universal constants.

The work of SB and SKG were supported in part by Council of Scientific and Industrial Research, Govt. of India.

\section{References}

[1] S. Chandrasekhar, An Introduction to the Study of Stellar Structure, Dover Publications, New York, 1958 and references therein.

[2] L. D. Landau, Phys. Z. Sowjetunion 1, 285 (1932), reprinted in Collected Works of L. D. Landau (Ed. D Ter Haar), p. 60, Pergamon Press, Oxford, 1965.

[3] S. L. Shapiro and S. A. Teukolsky, Black Holes, White Dwarfs and Neutron Stars, John Wiley \& Sons, New York, 1983.

[4] G. Baym and S. -A. Chin, Phys. Lett. B62, 241 (1976)

[5] C. Alcock, E. Farhi and A. Olinto, Ap. J. 310, 261 (1986) 
[6] See, for example, J. Madsen, in : Hadrons in dense matter and hadrosynthesis, Lecture Notes in Physics, Springer Verlag, Heidelberg (to appear); astro-ph/9809032 (1998)

[7] E. Witten, Phys. Rev. D30, 274 (1984)

[8] P. Haensel, J. L. Zdunik and R. Schaeffer, Astron. Astrophys. 160, 121 (1986)

[9] S. K. Ghosh and P. K. Sahu, Int. J. Mod. Phys. E2, 575 (1993)

[10] J. Alam, S. Raha and B. Sinha, Ap. J. 513, 572 (1999)

[11] D. Enstrom et al, astro-ph/9802236 (1998); S. Fredriksson et al, astroph/9810389 (1998)

[12] G. N. Fowler, S. Raha and R. M. Weiner, Z. Phys C9, 271 (1981); M. Plümer, Quark - Gluon - Plasma und vielfacherzeugung in der Starken Wechselwirkung, Doctoral dissertation, Philipps Universität, Marburg/Lahn, Germany (1984).

[13] A. Chodos et al, Phys. Rev. D9, 3471 (1974) 